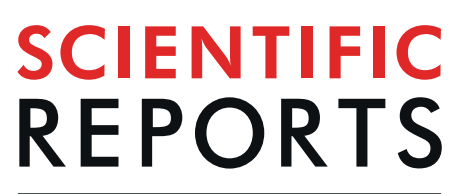

natureresearch

\title{
Fertilization and Cleavage Axes Differ In Primates Conceived By Conventional (IVF) Versus Intracytoplasmic Sperm Injection (ICSI)
}

\author{
Calvin R. Simerly ${ }^{1}$, Diana Takahashi ${ }^{2}$, Ethan Jacoby ${ }^{3}$, Carlos Castro ${ }^{1}$, Carrie Hartnett ${ }^{1}$, \\ Laura Hewitson ${ }^{4}$, Christopher Navara ${ }^{5}$ \& Gerald Schatten $\mathbb{~}^{1 *}$
}

With nearly ten million babies conceived globally, using assisted reproductive technologies, fundamental questions remain; e.g., How do the sperm and egg DNA unite? Does ICSI have consequences that IVF does not? Here, pronuclear and mitotic events in nonhuman primate zygotes leading to the establishment of polarity are investigated by multidimensional time-lapse video microscopy and immunocytochemistry. Multiplane videos after ICSI show atypical sperm head displacement beneath the oocyte cortex and eccentric para-tangential pronuclear alignment compared to IVF zygotes. Neither fertilization procedure generates incorporation cones. At first interphase, apposed pronuclei align obliquely to the animal-vegetal axis after ICSI, with asymmetric furrows assembling from the male pronucleus. Furrows form within $30^{\circ}$ of the animal pole, but typically, not through the ICSI injection site. Membrane flow drives polar bodies and the ICSI site into the furrow. Mitotic spindle imaging suggests para-tangential pronuclear orientation, which initiates random spindle axes and minimal spindle:cortex interactions. Parthenogenetic pronuclei drift centripetally and assemble astral spindles lacking cortical interactions, leading to random furrows through the animal pole. Conversely, androgenotes display cortex-only pronuclear interactions mimicking ICSI. First cleavage axis determination in primates involves dynamic cortex-microtubule interactions among male pronuclei, centrosomal microtubules, and the animal pole, but not the ICSI site.

With perhaps ten million ART babies now, fundamental problems regarding the mechanisms of fertilization and the onset of early development remain. Foremost among these are questions regarding which events are essential for the union of sperm and egg DNA within the activated zygote, as well as the consequences of different ART approaches, especially IVF as compared to ICSI, on the resultant embryo ${ }^{1-6}$. Notwithstanding the impressive literature on murine reproduction ${ }^{7-11}$, many differences between rodents and primates, especially in centriole and centrosome transmission ${ }^{12-15}$, preclude relevant conclusions. While human zygotes are investigated noninvasively, especially with the adoption of commercial time-lapse imaging systems, the earliest events during fertilization may not be captured in these systems, particularly during conception by ICSI. While research on animal models is increasingly regulated and has even been abandoned by some institutions (e.g., the UK's historic Sanger Institute $\left.{ }^{16}\right)$, responsible research, especially research mimicking the noninvasive procedures employed on

${ }^{1}$ Pittsburgh Development Center, Division of Developmental \& Regenerative Medicine, and Obstetrics-GynecologyReproductive Sciences, Cell Biology, and Bioengineering, University of Pittsburgh Cancer Institute, University of Pittsburgh School of Medicine, 204 Craft Avenue Pittsburgh, Pennsylvania, 15213, USA. ${ }^{2}$ Division of Cardiometabolic Health, Oregon National Primate Research Center, Oregon Health \& Science University, Beaverton, Oregon, 97006, USA. ${ }^{3}$ CCRM Houston Main Center Memorial City, 929 Gessner Rd, Suite 2300, Houston, Texas, 77024, USA. ${ }^{4}$ The Johnson Center for Child Health and Development, Austin, Texas, 78701, USA. ${ }^{5}$ Department of Biology, South Texas Center for Emerging Infectious Disease, University of Texas at San Antonio, San Antonio, Texas, 78249, USA. *email: schattengp@upmc.edu 
properly informed consenting couples undergoing infertility therapy, holds real promise for understanding basic principles during conception.

The establishment of embryonic axes that assemble the body's three-dimensional plan, crucial for body patterning, implantation, and perhaps, embryonic stem cell derivations (including therapeutic cloning), are not well understood in most mammals ${ }^{17-19}$. Features defining undeniable oocyte polarity in mammals, as observed in some vertebrate (e.g., frogs) and invertebrate eggs, were largely lacking until pioneering work from several laboratories provided compelling evidence that ingrained polarities in mouse oocytes, before and after fertilization, track closely with features of the mouse blastocyst, the first stage with clearly morphologically identifiable polarity ${ }^{20-24}$. Specifically, the first cleavage plane predictably aligned orthogonally to the embryonic-abembryonic axes of the bilaterally symmetrical blastocyst ${ }^{25}$. The second polar body $\left(2^{\text {nd }} \mathrm{PB}\right.$; the product of the maturation of an egg cell during chromosomal reductional divisions) is the fiduciary mark of the animal pole in the mouse zygote and marking experiments of this site have demonstrated a strong correlation between the first division plane and the animal-vegetal $(\mathrm{A} \rightarrow \mathrm{V})$ pole ${ }^{20,26,27}$. The sperm-induced fertilization cone may also be an accurate determinant of the cleavage plane ${ }^{28-31}$. Other experiments suggest cell shape, especially as influenced by the zona pellucida, is perhaps the most determinative factor for cleavage plane orientation ${ }^{32}$. Time-lapse video microscopy (TLVM) studies demonstrate how a pronounced oocyte shape change accompanying sperm incorporation mediated by actin influences the first cleavage axis, with the furrow passing through the short axis of the zygote and the site of sperm incorporation ${ }^{33}$. However, others question the assertion that the early mouse egg displays any predetermined axes. The orientation of the apposed male and female pronuclei was the most accurate predictor of the first cleavage plane since this axis defines the first mitotic spindle apparatus alignment, with cytokinesis occurring orthogonally to the aligned metaphase chromosomes ${ }^{34,35}$.

In humans, understanding zygotic polarity and embryonic axes relates to how best to select premier embryos at Day 3 or 5 for implantation and infertility management ${ }^{36}$. High-resolution time-lapse cinematography or the EmbryoScope ${ }^{\text {TM }}$ TLVM system have been utilized to investigate both ICSI- and IVF-derived zygotes ${ }^{37-41}$. The highest quality embryos are derived from human IVF or ICSI zygotes with polarized nucleoli that tend to divide early, while poorer quality human zygotes appear to have a significantly higher variation between pronuclear alignment and the $2^{\text {nd }} \mathrm{PB}^{42-49}$. In a retrospective time-lapse analysis of human ICSI zygotes, $95 \%$ underwent a meridional first cleavage division, demonstrating tetrahedral orientation at the 4-cell stage, predictive of the highest quality blastocysts for cyropreservation/embryo transfer ${ }^{50}$. Other TLVM human studies describe transient fertilization cone assembly (albeit at low frequency), pronuclei migration rates of $50 \mu \mathrm{m} / \mathrm{hr}$, and varying juxtapositioned pronuclei within the zygote's cytoplasm, but how these observations influence overall embryo quality is not yet known ${ }^{41}$. Ironically, in the rhesus monkey, an animal model closely aligned to human fertilization events ${ }^{13}$, fluorescent bead marking of ICSI sperm injection sites suggests that the sperm entry point (SEP) oriented the first cleavage plane and later aligned along the embryonic-abembryonic boundary in the blastocyst, as described for mice ${ }^{30}$.

To that end, this report presents findings on fertilization and early development in two primate models, macaques and baboons, with implications for understanding the still mysterious processes of human fertilization and the establishment of embryonic polarity.

Notwithstanding the impressive discoveries made by studying mice, clinically relevant findings rely upon studies on primates - human and nonhuman, alike ${ }^{13}$. Here, we have asked how similar ICSI is to conventional IVF. ICSI is perhaps the most used procedure in ART clinics; it is utilized extensively even in the absence of a defined male factor issue ${ }^{51}$. Consequently, this investigation focuses on fertilization and early developmental events in nonhuman primate (NHP) models to more accurately examine the events of human fertilization and how they might influence axis specifications with more clinical relevance.

\section{Results}

IVF- and ICSI-Fertilized zygotes differ in the cytoplasmic pronuclear orientation patterns after migration. To investigate early pronuclear events in IVF- and ICSI-fertilized zygotes, we performed multidimensional TLVM recordings after fertilization (Figs 1 and 2; Supplemental Videos 1-3). A fused, anchored sperm head, bound tightly to the oolemma by 3 hrs post-IVF, is depicted in Fig. 1A1-A6. Cortical rotation, first toward the site of the arrested second meiotic spindle, followed by a gradual return to a distal cortical site, was indictive of the oocyte shape changes during the first $13 \mathrm{hrs}$ post-insemination (Supplemental Videos 1). A female pronucleus (FPn) formed after oocyte activation, eventually moving toward the cortically residing sperm head (Fig. 1A7-A12), presumably along a microtubule-based sperm aster assembled from the reconstituted centrosomes ${ }^{12,52,53}$. No sperm incorporation cone assembled, though cortical streaming was evident (Supplemental Video 1). A second IVF-inseminated rhesus TLVM recording showed that the male pronucleus (MPn) formed at the oolemma sperm penetration site (Fig. 1B1-B6), with little cortical rotation, but with cytoplasmic contraction-expansion cycles observed (Supplemental Video 2). FPn migration to the cortical MPn measured $0.387 \pm 0.056 \mu \mathrm{m} \mathrm{min}^{-1}(\mathrm{n}=5$; Fig. 1B7-B12), with all zygotes showing radially apposed pronuclei relative to the adjacent overlying cortex and $80 \%$ aligning obliquely to the $\mathrm{A} \rightarrow \mathrm{V}$ axis by late interphase (Table 1; Supplemental Fig. S1).

Rhesus ICSI oocyte TLVM recordings are presented in Fig. 2 and Supplemental Video 3. An immobilized spermatozoon microinjected near the cortex showed significant cytoplasmic sperm head displacement compared to early attached IVF sperm, presumably derived from cytoplasmic streaming forces ${ }^{54}$, before MPn cortical formation (Fig. 2A1-A6). Following oocyte activation and $2^{\text {nd }} \mathrm{PB}$ extrusion, the FPn formed at the animal pole before migrating rapidly toward the MPn along presumed assembled sperm astral microtubules (Fig. 2A7-A12 ${ }^{55}$ ). FPn migration rates (Table 1) measured at $0.539 \pm 0.044 \mu \mathrm{m} \mathrm{min}^{-1}(\mathrm{n}=48)$ compared to IVF migration rates of $0.387 \pm 0.056(\mathrm{n}=5$; not significant at $\mathrm{p}<0.27)$. ICSI apposed pronuclei aligned mostly para-tangentially to the adjacent cortex $(58 \% ; \mathrm{n}=52)$ but also obliquely to the $\mathrm{A} \rightarrow \mathrm{V}$ axis $(86 \% ; \mathrm{n}=52$; Table 1$)$. Conversely, ICSI in the oocyte's central cytoplasm showed decondensing MPn moving by cytoplasmic streaming within the 

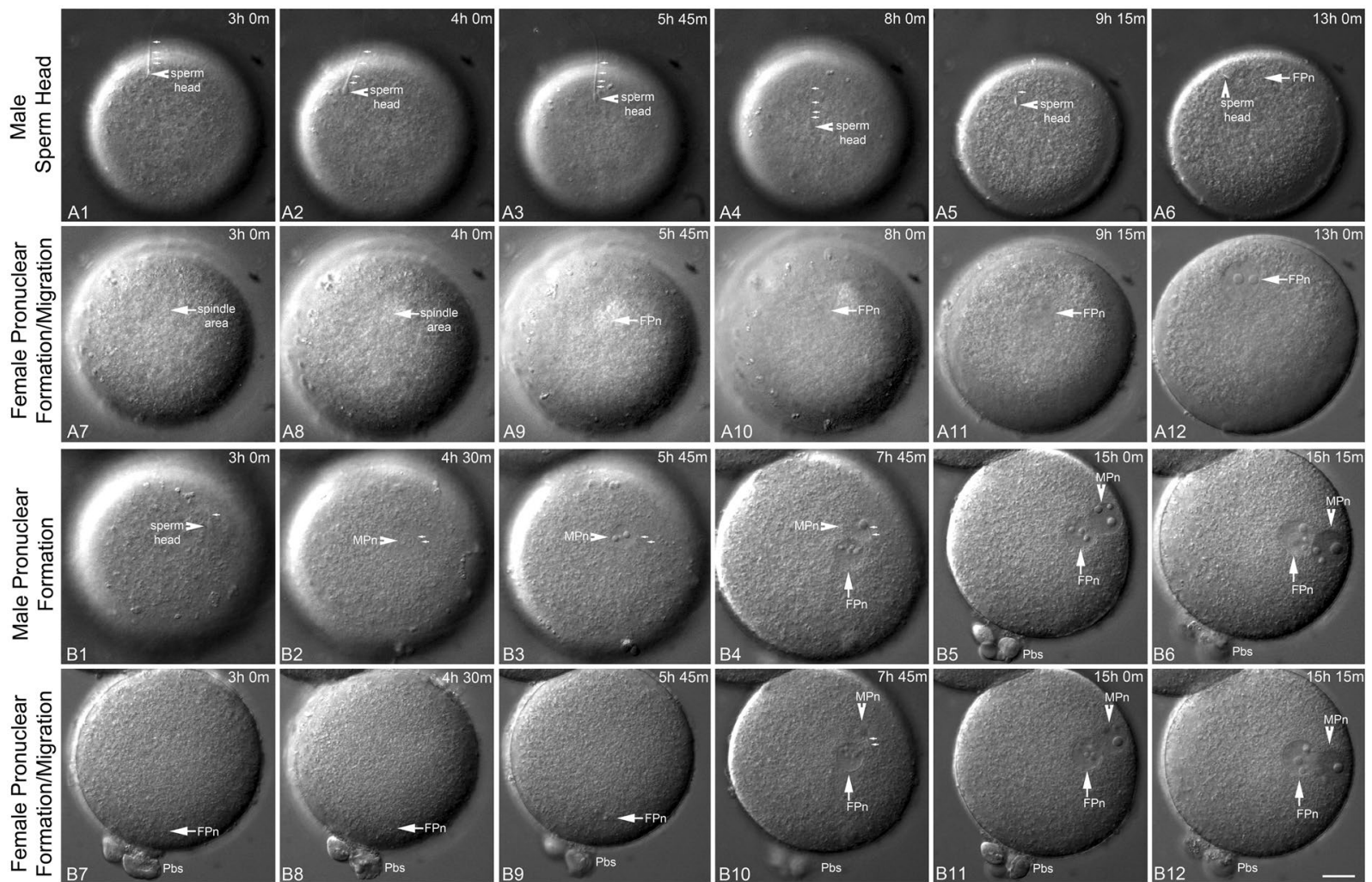

Figure 1. Selected TLVM panels of Rhesus sperm incorporation and pronuclear apposition during IVF. (A1-A6): male sperm head events during IVF (arrowheads) showing incorporation (A1,A2) followed by cortical rotation, first toward the site of the oocyte's spindle region (A3-A4) and subsequently to a distal cortical site (A5-A6). The sperm head shows firm attachment to the oocyte's cortex during penetration, even as cortical rotation powers sperm translocation. The sperm head is delayed in conversion to a fully decondensed MPn. (A7-A12): corresponding image panels from the TLVM video showing the position of the second meiotic spindle (A7-A8, arrows), FPn formation (A9-A11, arrows) and migration to the cortically residing sperm head (A12, arrow), mediated by the assembled sperm aster. Extrusion of the $2^{\text {nd }} \mathrm{PB}$ is not visible in these panels. (B1-B12). Classical rhesus IVF and pronuclear apposition beginning $3 \mathrm{hrs}$ post-insemination show sperm head plasma membrane penetration (B1, arrowhead), MPn formation (B2-B3, arrowheads), male and female pronuclear apposition (B4: MPn, arrowhead; FPn, arrow), and typical radial cortical parental genome orientation by the middle of interphase, with the MPn closest to the oocyte's cortex (B5-B6, arrowheads) and the female distal within the cytoplasm (B5-B6, arrows). Note the orientation of the apposed pronuclei is oblique to the $\mathrm{A} \rightarrow \mathrm{V}$ axis. (B7-B12): corresponding images of FPn events. After $2^{\text {nd }} \mathrm{PB}$ extrusion by $3 \mathrm{hrs}$ post-insemination, the FPn forms near the cortex (B7-B9, arrows), swiftly migrates to the MPn utilizing microtubules of the assembled sperm aster (B10-B11, arrows), and ultimately positions distal to the cortically residing MPn (B12; FPn: arrow; MPn: arrowhead). The small arrows trace the sperm axoneme in

(A1-A5,B1-B4). Time is given in hours:minutes (h:m) post-IVF. All panels represent selected HMC images from a TLVM recording. Bar $=20 \mu \mathrm{m}$.

\begin{tabular}{|c|c|c|c|c|c|c|c|c|}
\hline \multirow[b]{2}{*}{$\begin{array}{l}\text { Insemination } \\
\text { type }\end{array}$} & \multirow[b]{2}{*}{$\begin{array}{l}\text { Total n } \\
\text { scored }\end{array}$} & \multirow{2}{*}{$\begin{array}{l}\text { Oocyte } \\
\text { insemination } \\
\text { position }\end{array}$} & \multirow{2}{*}{$\begin{array}{l}\text { Mean } \pm \text { S.E. rate of } \\
\text { o PN migration to } \sigma^{x} \\
\mathrm{PN} \text { in } \mu \mathrm{m} \mathrm{min} \mathbf{m i n}^{-1}[\mathrm{n}]\end{array}$} & \multicolumn{2}{|c|}{$\begin{array}{l}\text { Pronuclear Orientation to } \\
\text { Oolemma (\%) }\end{array}$} & \multicolumn{3}{|c|}{$\begin{array}{l}\text { Pronuclear Alignment Relative to Oocyte } \\
\text { Axis at NEBD (\%) }\end{array}$} \\
\hline & & & & radial & $\begin{array}{l}\text { Para- } \\
\text { tangential }\end{array}$ & $\begin{array}{l}\text { Meridional } \\
\text { axis }(\mathbf{A} \rightarrow \mathbf{V})\end{array}$ & $\begin{array}{l}\text { Equatorial } \\
\text { axis }\end{array}$ & $\begin{array}{l}\text { Oblique } \\
\text { axis }\end{array}$ \\
\hline IVF & 5 & cortical & $0.387 \pm 0.056[5]$ & $5 / 5(100)$ & 0 & 0 & $1 / 5(20)$ & $4 / 5(80)$ \\
\hline ICSI & 52 & cortical & $0.539 \pm 0.044^{\mathrm{a}, \mathrm{b}}[35]$ & $22 / 52(42)$ & $30 / 52(58)$ & $3 / 52(6)$ & $4 / 52(8)$ & $45 / 52(86)$ \\
\hline ICSI & 4 & central & $0.688 \pm 0.118^{c}[4]$ & $1 / 4(25)$ & $3 / 4(75)$ & 0 & $1 / 4(25)$ & $3 / 4(75)$ \\
\hline
\end{tabular}

Table 1. NHP Pronuclear Migration, Cortical Alignment and Orientation to Zygotic Axes Analyzed from TLVM Recordings. ${ }^{a}$ female pronuclear migrate rate not significantly different between ICSI cortical versus IVF cortical sperm $(\mathrm{p}<0.27)$. ${ }^{b}$ female pronuclear migration rate not significantly different between ICSI cortical versus ICSI central sperm $(\mathrm{p}<0.61)$. ${ }^{c}$ female pronuclear migration rate not significantly different between ICSI central versus IVF cortical sperm $(\mathrm{p}<0.16)$. 


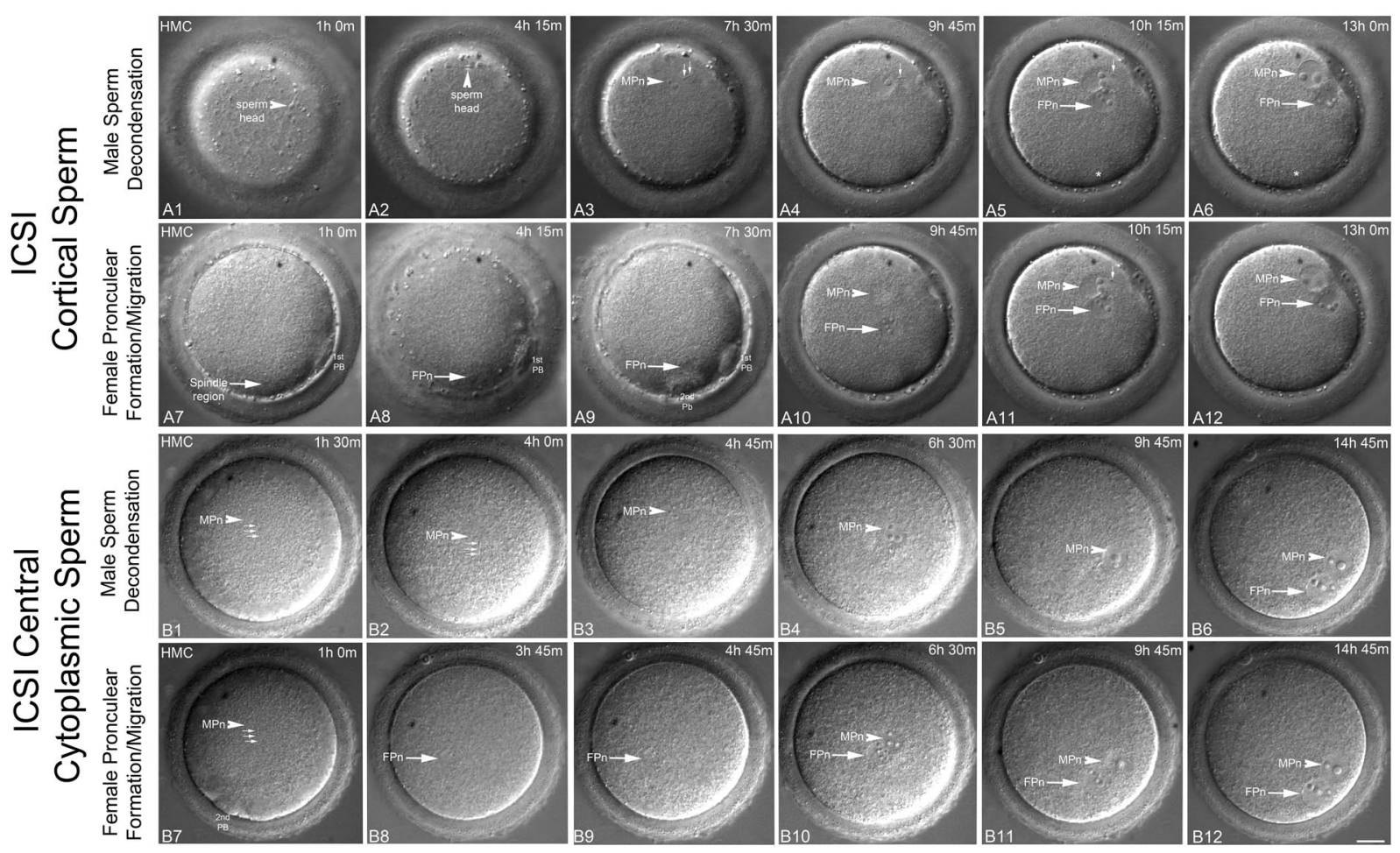

Figure 2. Selected TLVM panels of NHP sperm head decondensation, pronuclear assembly, migration and orientation following ICSI. (A1-A12). FPn and MPn formation, apposition, and cytoplasmic orientation by mid-interphase. Following ICSI, the cortically residing sperm head is visible translocating along the cortex, probably by cytoplasmic flow (A1-A2, arrowheads). Sperm head decondensation into an MPn is observed cortically (A3-A4, arrowheads) as the sperm aster assembles and promotes FPn migration (A5: MPn, arrowhead; FPn, arrow). Apposed FPn and MPn are oriented para-tangentially to the overlying cortex and obliquely to the $\mathrm{A} \rightarrow \mathrm{V}$ axis (A6: MPn, arrowhead; FPn, arrow; $2^{\text {nd }} \mathrm{PB}$ position out of focal plane marked with *). FPn formation following elicitation of the second polar $\left(2^{\text {nd }} \mathrm{PB}\right)$ is observed in (A7-A9) (arrows) with migration to the cortical MPn and tangential positioning at the cortex seen in A10-A12 (arrows; FPn, female pronucleus; MPn, male pronucleus). (B1-B12). FPn and MPn events after sperm deposition in the central cytoplasm. After central cytoplasmic sperm deposition by ICSI, the sperm head undergoes decondensation (B1-B2, arrowheads; small arrows, incorporated sperm axoneme) as the developing MPn jostles within the central cytoplasm (B2-B4; arrowheads). The FPn migrates along sperm astral microtubules to arrive at the MPn (B4, arrowhead) and the apposed parental genomes translocate to the oolemma cortex, aligning paratangentially to the zygote surface and obliquely to the $\mathrm{A} \rightarrow \mathrm{V}$ axis $\left(2^{\text {nd }} \mathrm{PB}\right.$ in B7). FPn forms after $2^{\text {nd }} \mathrm{PB}$ formation (B7-B9: arrows) and completes migration to the decondensed MPn by $6.5 \mathrm{hr}$ post insemination (B10: FPN, arrow; MPn, arrowhead). Over the next $8 \mathrm{hrs}$, the apposed parental genomes translocate to the cortex (B11-B12; FPn, arrow; MPn, arrowhead). The small arrows trace the sperm axoneme in (A3A5,A11,B1,B2,B7). Time is given in hours:minutes (h:m) post ICSI. All panels represent selected HMC images from a TLVM recording. Bar $=20 \mu \mathrm{m}$.

cytoplasm, translocating to the cortex only after FPn apposition (Fig. 2B1-B6). FPn migration rates measured $0.688 \pm 0.118 \mu \mathrm{m} \mathrm{min}{ }^{1}$ (Table $1 ; \mathrm{n}=4$ ), with shorter migration distances. The apposed pronuclei largely aligned para-tangentially to the overlying cortex and obliquely to the $\mathrm{A} \rightarrow \mathrm{V}$ axis after migration $(75 \% ; \mathrm{n}=4$; Table 1$)$.

Taken together, multidimensional TLVM observations of ICSI rhesus zygotes showed extensive cortical spermatozoon displacement and unique para-tangential pronuclear alignment relative to the overlying cortex, but not the $\mathrm{A} \rightarrow \mathrm{V}$ axis, compared to IVF-fertilized zygotes. Volumetric changes in the shape of zygotes, along with cytoplasmic streaming forces, might influence the cortical pronuclear para-tangential alignment crucial to cleavage plane development at mitosis.

The ICSI zygote asymmetric cleavage furrow originates near the cortical male pronuclear breakdown site, progressing though the animal pole, but not the sperm entrance site. In mice, the first cleavage plane may not be random but dependent on being parallel to the line of pronuclear apposition and within $30^{\circ}$ of the second polar body and sperm incorporation site ${ }^{26}$. Alternatively, mouse zygotes may cleave perpendicularly to the plane of the approaching male and female pronuclei, irrespective of the $2^{\text {nd }} \mathrm{PB}$ or sperm incorporation site, or orthogonally to the bilaterally symmetrical zygote, regardless of the $2^{\text {nd }} \mathrm{PB}$, sperm incorporation site, or pronuclear orientation ${ }^{27,56,57}$. 


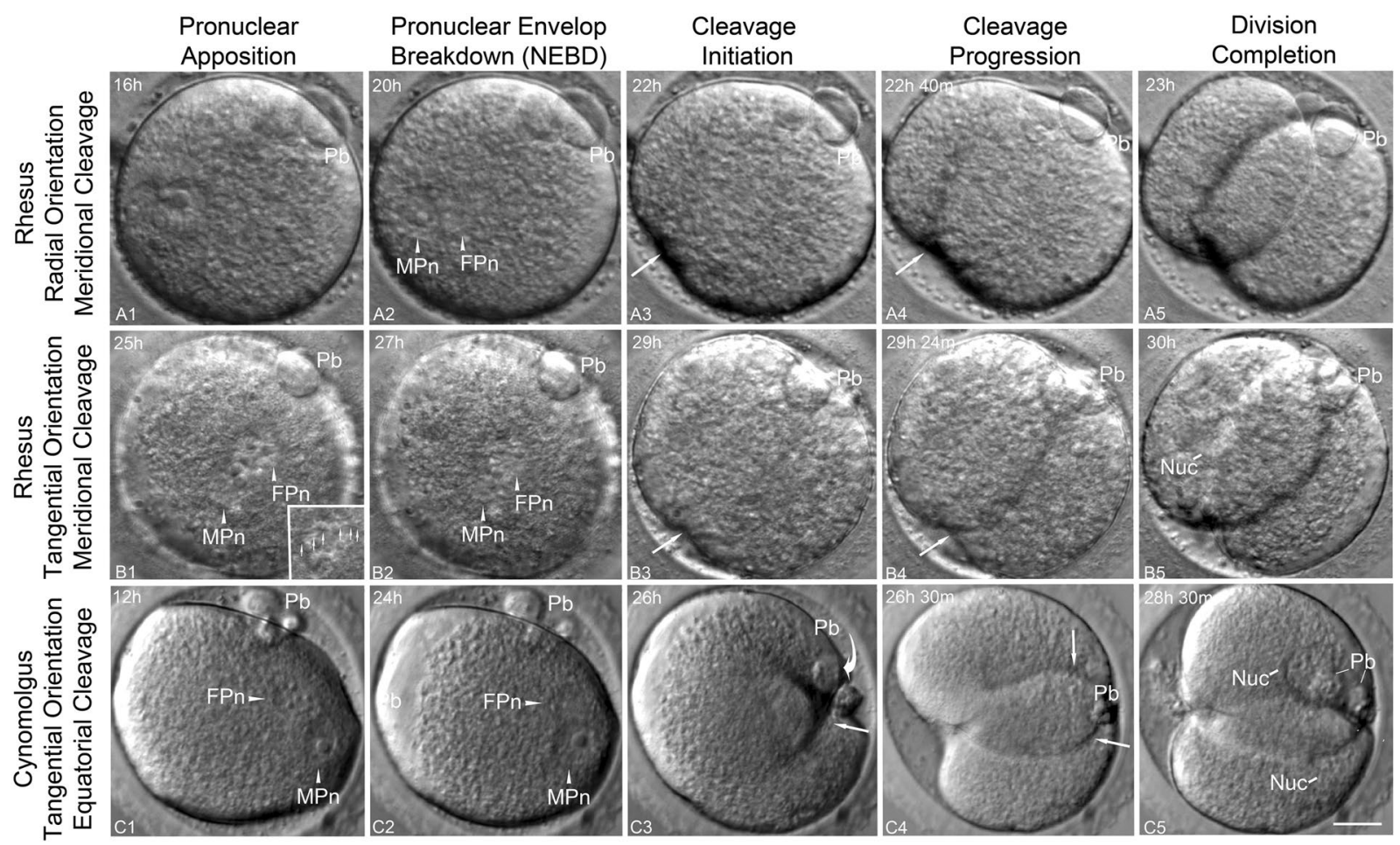

Figure 3. Cleavage furrow initiation and zygotic division in NHP zygotes with varying pronuclear orientations. (A1-A5) Rhesus zygote showing radially aligned apposed pronuclei with oblique orientation to the $\mathrm{A} \rightarrow \mathrm{V}$ axis $16 \mathrm{hrs}$ post-ICSI (A1; polar body, $\mathrm{Pb}$ ). Female pronuclear breakdown occurs first, around $20 \mathrm{hrs}$ postICSI (A2). A meridional cleavage begins at the cortex overlying the site of the formerly intact MPn (A3: arrow), progressing asymmetrically through the cytoplasm to within $30^{\circ}$ of the second polar body $(\mathrm{Pb})$ on the opposing cortex (A4: arrow) $22 \mathrm{~h}$ post-ICSI. After division at $23 \mathrm{hrs}$ post-ICSI, both $\mathrm{Pb}$ reside between the daughter blastomeres (A5). (B1-B5) A rhesus zygote with para-tangentially aligned cortical parental genomes oriented along the $\mathrm{A} \rightarrow \mathrm{V}$ axis 25 hrs post-ICSI (B1; polar body, $\mathrm{Pb}$; inset, details of apposed pronucleus and the incorporated sperm axoneme, small arrows). At $27 \mathrm{hrs}$ post-ICSI, pronuclear breakdown begins with the MPn (B2; MPn). Cleavage furrow initiation (B3: arrow) begins at the cortex overlying the site of the formerly intact MPn, progressing quickly through a meridional plane that includes the second polar body (B4: $\mathrm{Pb}$ ). Cytokinesis at $30 \mathrm{hrs}$ post-ICSI creates equal-sized daughter blastomeres with the polar bodies residing between the cleaved cells (B5: Pb; Nuc: daughter cell nucleus). (C1-C5) A cynomolgus ICSI zygote at $12 \mathrm{hrs}$ post-ICSI showing apposed parental genomes aligned para-tangentially to the cortex and obliquely to the $\mathrm{A} \rightarrow \mathrm{V}$ axis $(\mathbf{C 1})$. The FPn undergoes breakdown first at $24 \mathrm{hrs}$ post-ICSI (C2). However, cleavage furrow initiation at $26 \mathrm{hrs}$ post-ICSI moves in an equatorial direction or right angle to the $\mathrm{A} \rightarrow \mathrm{V}$ axis (C3, straight arrow) while cortical membrane flow rotates both polar bodies into the developing furrow $(\mathbf{C 3}, \mathrm{Pb}$, curved arrow depicts rotation direction). The bifurcated cleavage furrow progresses towards the opposite cortex (C4; arrows) as cytokinesis forms equal-sized daughter blastomeres with the polar bodies between daughter cells by $28.5 \mathrm{hrs}$ post-ICSI (C5: polar bodies, Pb; Nuc: reconstituted daughter cell nuclei). All panels are HMC images taken from TLVM videos of ICSI fertilized zygotes undergoing first cleavage. Time in hours:minutes (h:m) post-ICSI. Bar $=20 \mu \mathrm{m}$.

\begin{tabular}{|c|c|c|c|c|c|c|c|c|c|c|c|c|c|}
\hline \multirow[t]{2}{*}{$\begin{array}{l}\text { Insem } \\
\text { type }\end{array}$} & \multirow[t]{2}{*}{$\begin{array}{l}\mathbf{N} \\
\text { scored }\end{array}$} & \multirow[t]{2}{*}{\begin{tabular}{|l|} 
Sperm MIP and \\
pronuclear orientation \\
to overlying cortex at \\
NEBD \\
\end{tabular}} & \multicolumn{3}{|c|}{$\begin{array}{l}\text { Cleavage initiation at last } \\
\text { position of: }(\%)\end{array}$} & \multicolumn{3}{|c|}{$\begin{array}{l}\text { Cleavage furrow angle relative } \\
\text { to the animal pole }\left[2^{\text {nd }} P B\right](\%)\end{array}$} & \multicolumn{2}{|c|}{ Cleavage type (\%) } & \multirow[t]{2}{*}{\begin{tabular}{|l|}
$2^{\text {nd }}$ PB between \\
daughter \\
blastomeres post \\
division (\%)
\end{tabular}} & \multicolumn{2}{|c|}{$\begin{array}{l}\text { Blastomere size post } \\
\text { division }(\%)\end{array}$} \\
\hline & & & PN & PN & Other $^{\mathrm{a}}$ & $0-30^{\circ}$ & $31-60^{\circ}$ & $>60^{\circ}$ & Mer & Eq & & Equal & Not Equal \\
\hline ICSI & 19 & cortical radial & $15 / 19(79)$ & $1 / 19(5)$ & $3 / 19(16)$ & $13 / 19(68)$ & $4 / 19(21)$ & $2 / 19(11)$ & $13 / 19(68)$ & $6 / 19(32)$ & $16 / 19(84)$ & $7 / 19(37)$ & $12 / 19(63)$ \\
\hline ICSI & 37 & cortical para-tangential & $25 / 37(68)$ & 0 & $12 / 37(32)$ & $26 / 37(70)$ & $5 / 37(14)$ & $6 / 37(16)$ & $26 / 37(70)$ & $11 / 37(30)$ & $31 / 37(84)$ & $15 / 37(41)$ & $22 / 37(59)$ \\
\hline Partho & 5 & NA & NA & 0 & $5 / 5(100)$ & $3 / 5(60)$ & $2 / 5(40)$ & 0 & $3 / 5(60)$ & $2 / 5(40)$ & $5 / 5(100)$ & $4 / 5(80)$ & $1 / 5(20)$ \\
\hline
\end{tabular}

Table 2. NHP Zygote Cleavage Patterns in Relation to Pronuclear Cortical Orientation and the Zygotic $\mathrm{A} \rightarrow \mathrm{V}$ Axis Analyzed from TLVM Recordings. Insem: insemination; Mer: meridional cleavage; Eq: equatorial cleavage; NEBD: pronuclear envelop breakdown; Partho: parthenogenetic chemical activation by ionomycin/6-DMAP

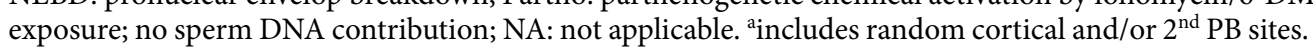




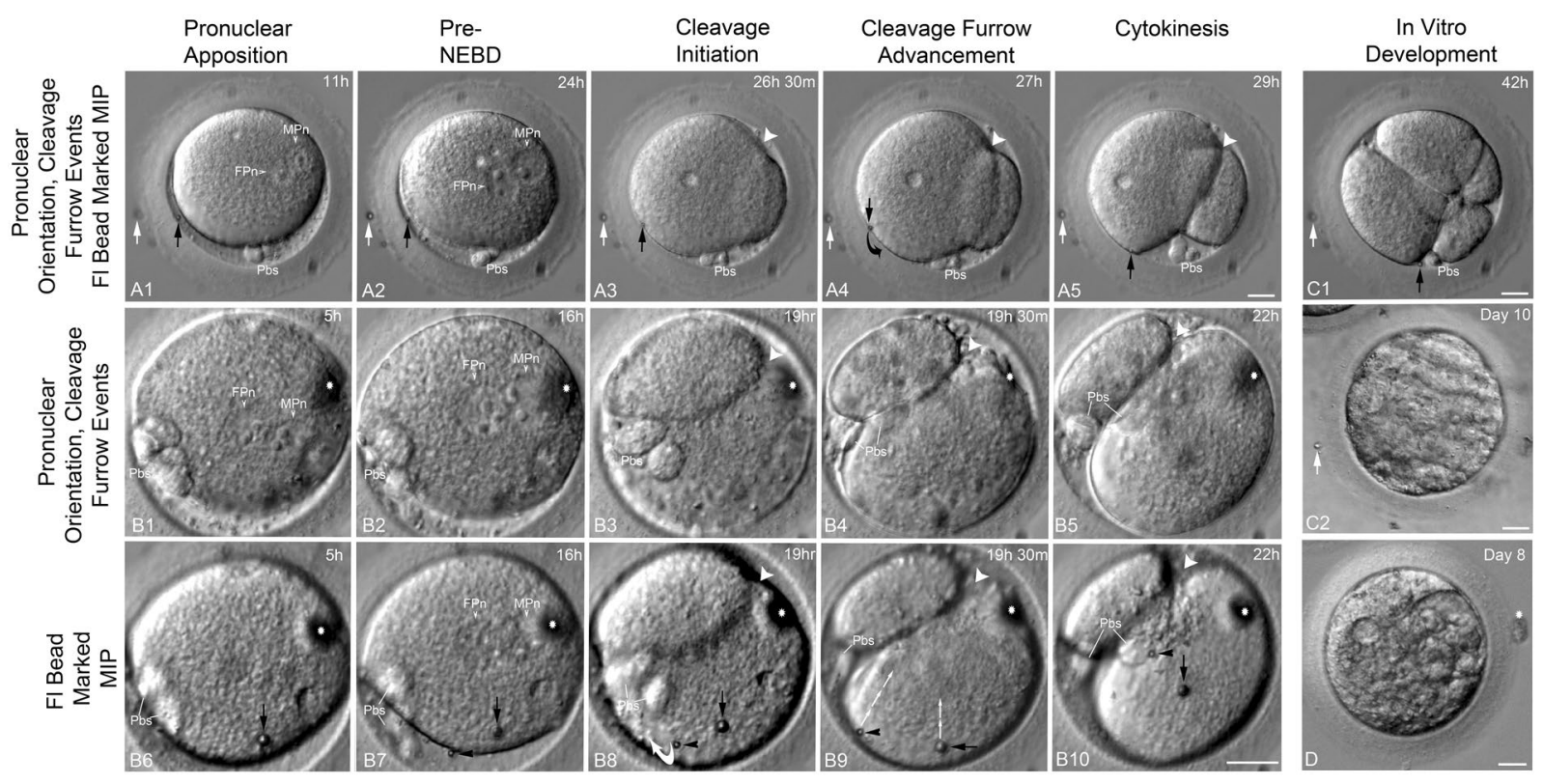

Figure 4. Neither cleavage furrow initiation nor progression includes passage through the sperm microinjection point (MIP). (A1-A5) A Baboon zygote with radially aligned apposed pronuclei oriented obliquely to the $\mathrm{A} \rightarrow \mathrm{V}$ zygotic axis. A detached 3-micron bead within the perivitelline space marks the MIP (A1, black arrow). Before mitosis, the expanding cytoplasm re-engages the bead (A2, black arrow). An asymmetric cleavage furrow forms near the previously intact apposed pronuclei (A3, large arrowhead), progressing meridionally through the polar bodies (Pbs), but not the MIP (A3, black arrow). The attached bead rotates by surface membrane flow toward the furrow (A3-A4, black arrows). Post-cytokinesis, the embryo shows unequal cleavage, with polar bodies in the division plane and the bead near the furrow (A5, black arrow). (B1-B10). A Baboon zygote showing pronuclear events (B1-B5) and movement of two attached marking beads during cytokinesis (B6-B10). A 5-micron bead marks the MIP (B6, black arrow); the 3-micron bead marks a distal cortex site (B7, black arrowhead). Radially aligned apposed pronuclei are oblique to the $\mathrm{A} \rightarrow \mathrm{V}$ axis (B1) with a bead-marked MIP (B6: black arrow). The apposed pronuclei translocate cortically, aligning with the $\mathrm{A} \rightarrow \mathrm{V}$ axis pre-mitosis (B2). Slight membrane flow exposes both attached beads (B7, black arrow, arrowhead). Furrow initiation occurs near the formerly intact MPn (B3, white arrowhead), proceeding meridionally toward the polar bodies (Pbs), but not through the beads (B8, black arrow, arrowhead). Beads show independent movement; the 3-micron bead initially toward the polar bodies (B8, white curved arrow) before redirecting toward the furrow, while the 5-micron MIP bead moves vertically toward the mid-furrow (B9, small white arrows). Cytokinesis is unequal, with polar bodies (Pbs) between blastomeres and both beads near the furrow (B10, black arrow, arrowhead). (C1-C2). The A1-A5 Baboon zygote after in vitro development. Second division is equatorial in both daughter cells, with the MIP bead near the polar body between blastomeres (C1; black arrow). (D) The B1-B10 baboon blastocyst on Day 8 post-in vitro culture. All panels are HMC images. Time (hours:minutes) post-ICSI. Vac: cytoplasmic vacuole(s). White arrow: zona pellucida reference bead; White *zona pellucida surface debris. Bars $=20 \mu \mathrm{m}$.

\begin{tabular}{|l|l|l|l|l|l|}
\hline & \multicolumn{5}{|c|}{$\begin{array}{l}\text { Cleavage Furrow Initiation and Progress in relation to the MIP site marked } \\
\text { by a Fluorescent Bead }\end{array}$} \\
\cline { 3 - 6 } $\begin{array}{l}\text { Sperm MIP/Cortical } \\
\begin{array}{l}\text { Pronuclear Orientation } \\
\text { to A } \rightarrow \text { V axis }\end{array}\end{array}$ & $\begin{array}{l}\text { Total } \\
\text { Zygotes } \\
\text { Scored }\end{array}$ & $\begin{array}{l}\text { initiation } \\
\text { at the MIP } \\
(\%)\end{array}$ & $\begin{array}{l}\text { progression } \\
\text { through the } \\
\text { MIP (\%) }\end{array}$ & $\begin{array}{l}\text { both initiation and } \\
\text { progression through } \\
\text { the MIP (\%) }\end{array}$ & $\begin{array}{l}\text { Neither initiation or } \\
\text { progression through } \\
\text { the MIP (\%) }\end{array}$ \\
\hline Cortical/radial & 7 & 0 & $1 / 7(14)$ & $1 / 7(14)$ & $5 / 7(71)$ \\
\hline Cortical/para-tangential & 7 & 0 & $2 / 7(29)$ & $1 / 7(14)$ & $4 / 7(57)$ \\
\hline
\end{tabular}

Table 3. NHP Zygotes Do Not Initiate or Progress the Cleavage Furrow through the ICSI Microinjection Point (MIP).

We investigated cleavage plane initiation and progression by multidimensional TLVM in ICSI NHP zygotes undergoing first cell division (Fig. 3; Supplemental Videos 4, 5). Apposed radial (Fig. 3A1) or para-tangential (B1, C1) pronuclei began nuclear envelope breakdown (NEBD) 20-27 hrs post-ICSI, beginning with the FPn (36/52; $69 \%$ ), with the MPn 16-20 min later (Fig. 3A2,B2,C2). Cleavage furrow initiation began cortically, at the cortex closest to where the formerly intact MPn was last observed (Fig. 3A3,B3,C3) and progressing toward the $2^{\text {nd }} \mathrm{PB}$ (Fig. 3A4,B4). Cleavage furrow progression nearly always aligned to the animal pole (Table 2), moving diagonally across the zygote and sweeping the polar bodies into the cleavage furrow (Fig. 3C3). After division, equivalent 
blastomeres formed, with the polar bodies lying between the daughter cells, indicating a meridional cleavage (Fig. 3A5,B5,C5).

Table 2 summarizes NHP radial versus para-tangential pronuclear alignment with cleavage patterns. Regardless of pronuclear orientation, cleavage overwhelmingly initiated at the cortex adjacent to the last position of the intact MPn, passing within $30^{\circ}$ of the animal poles (meridional cleavage), with the $2^{\text {nd }} \mathrm{PB}$ lying between daughter cells after division. However, cell division after ICSI did not always result in equal-size daughter blastomeres (37-41\%; Table 2), and cytoplasmic fragmentation was notable.

Investigations on cleavage initiation and progression in relation to marked ICSI microinjection points (MIPs) using a phytohemagglutinin-labeled 3- to $5-\mu \mathrm{m}$ fluorescent bead is shown in Fig. 4 and Table 3. In most cases, whether apposed pronuclei were radially or para-tangentially oriented, the cleavage furrow neither initiated at, nor progressed through, the MIP (Table 3). The fluorescent beads marking the MIP site moved toward cleavage furrows owing to cortical rotation (Fig. 4A1-A5,B1-B10; Supplemental Videos 6, 7). Cleavage was consistently unequal after first division, although in vitro development to morula or early blastocyst stages appeared uncompromised (Fig. 3C1,C2,D).

Collectively, first division in ICSI zygotes begins asymmetrically at the cortex nearest where the intact MPn was last observed and not the sperm injection site. Cleavage progression is mostly toward the animal pole $\left(2^{\text {nd }} P B\right)$ in a meridional pattern. Cortical rotation translocates both the polar bodies and the MIP into the furrow region, consistent with a previous report describing the sperm injection site as localized near the first cleavage plane after cell division ${ }^{30}$.

Spindle orientation and microtubule:cortex interactions during division differ between IVFand ICSI-fertilized zygotes. In mice, acentriolar maternal microtubule organizing centers (MTOCs) nucleate cytoplasmic microtubules that "direct" pronuclear apposition to the oocyte's center, forming the centralized acentriolar, anastral, barrel-shaped bipolar mitotic spindles (Supplemental Fig. S2 ${ }^{12}$ ). Mitotic chromosome segregation is concomitant with an elongating spindle apparatus, crisscrossing opposite-pole microtubules, and a symmetrical invaginating furrow mediated by myosin II, the microfilament-associated motor protein involved in cleavage furrow activity ${ }^{58}$.

Unlike mice, other mammals, including humans and NHPs, inherit the dominant MTOC from the sperm (the paternal centrosome) to assemble the anastral bipolar mitotic metaphase spindle (Fig. $5^{12,52}$ ). The paternal centrioles in most mammals include the resurrection of a highly atypical distal centriole that undergoes duplication during interphase to provide the basis for mitotic bipolar spindle assembly ${ }^{14,15}$. The proximal sperm centriole remains with the incorporated sperm axoneme found on the paternal pronucleus, and by late interphase, the now duplicated paternal centrioles locate at the leading edges of the overlapping apposed pronuclei ${ }^{52}$. The mitotic spindle has one pole that retains the proximal centriole attached to the sperm tail and one pole with the resurrected distal centriole ${ }^{14}$.

In NHP zygotes, pronuclear orientation impacts cytoplasmic bipolar spindle orientation after NEBD (Fig. 5; Supplemental Fig. S3). For IVF-fertilized zygotes at late interphase, radially oriented pronuclei tightly associate with the adjacent cortex, and the centrioles mark the poles of assembled para-tangential spindles (Fig. 5A-C). Male and female chromosomes condense separately, align at the metaphase spindle equator, and division proceeds orthogonally to chromosome separation (Fig. 5C). At anaphase, spindle microtubules form transient overlapping bundles at the adjacent cortex, signaling asymmetric cleavage furrow formation (Fig. 5D, arrow; 5/5 IVF anaphase spindles). Midbody microtubules anchor the spindle apparatus to the invaginating furrow as it moves toward the opposing cortex (Fig. 5E). This pattern of spindle assembly and cleavage initiation favors equal daughter cell formation after cytokinesis $(15 / 23 ; 65 \%)$.

ICSI zygotes have more random pronuclear orientations (Table 1). Para-tangential pronuclear orientation positions centrioles favoring radially aligned mitotic spindles relative to the adjacent cortex (Fig. 5F-J; Supplemental Fig. S3). Observationally, assembled ICSI mitotic spindles appear at greater distances from the overlying cortex, with reduced or absent microtubule:cortex interactions (Fig. 5I,K1-K4,L1-L4; 2/14;14\%) that may cause cell division errors. However, ICSI zygotes form asymmetric cleavage furrows that pass orthogonally to segregating chromatids at anaphase but with more unequal cleavage divisions (Table 2; 25/42; 60\%).

Cytoplasmic pronuclear orientation, cleavage patterns, and microtubule configurations in parthenogenotes and androgenotes demonstrate that cortical affinity lies with the male pronucleus. Understanding of male and female pronuclear orientation benefits from studying parthenogenotes (no male contribution) and androgenotes (male contribution only). Parthenogenotes, created either by chemical treatment with ionomycin/6-DMAP or by injecting metaphase-II arrested oocytes with a crude sperm extract, form haploid or diploid female pronuclei that migrate randomly within the central cytoplasm (Supplemental Fig. S4). Conversely, androgenotes, created by injection of a single sperm into an "enucleated" oocyte (meiotic spindle:chromosome complex removed by a micropipette), form a single, cortex-associated MPn (Supplemental Fig. S4). A strong correlation exists between a decondensed MPn and eccentric cortical pronuclear positioning in NHP oocytes, although how this is initiated and maintained is not currently known.

TLVM recordings of parthenogenote and androgenote zygotes undergoing first mitosis are shown in Supplemental Fig. S5 and Supplemental Video 8. For parthenogenotes, mitotic spindle assembly occurred within the central cytoplasm after FPn breakdown, with an asymmetric cleavage furrow assembling from either the animal pole or a random cortical site (Table 2). Androgenotes, with the MPn closely associated with the overlying cortex, assembled cortical spindles and asymmetric cleavage furrows at the adjacent overlying cortex as observed for ICSI-fertilized zygotes (Supplemental Fig. S5; Supplemental Video 8). 

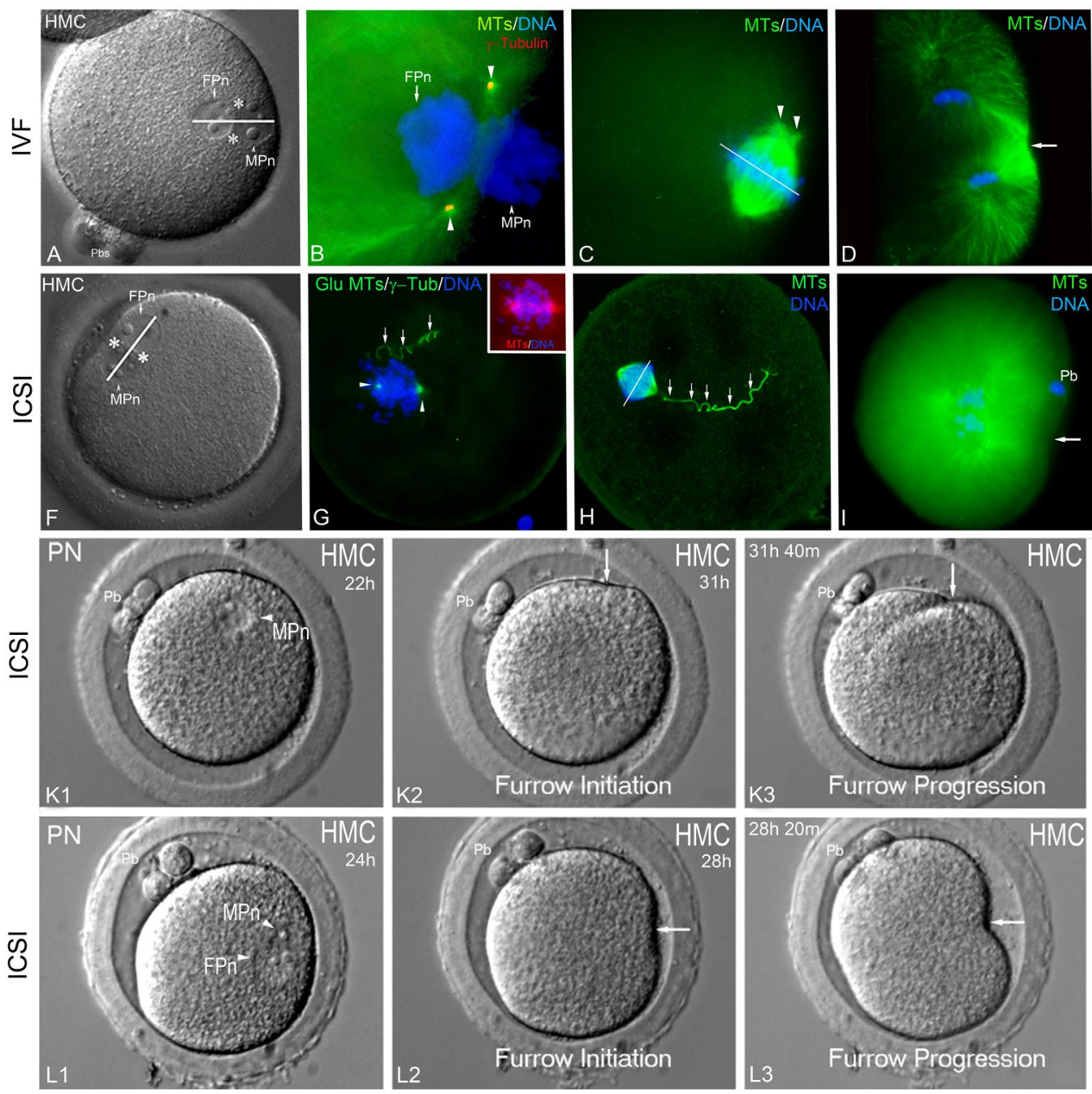

Figure 5. Mitotic spindle assembly and first cleavage patterns differ between IVF- and ICSI-Fertilized NHP Zygotes. Rhesus IVF- (A-E) or ICSI- (F-J) fertilized zygotes. (A,F) Interphase zygotes with radially oriented (IVF: A) versus para-tangentially (ICSI: F) aligned apposed male and female pronuclei. White asterisks: duplicated centrosome positions; white bars: spindle equator chromosome alignment and anaphase cleavage plane direction. $(\mathbf{B}, \mathbf{G})$ fixed immunostained zygotes with duplicated, split centrosomes (arrowheads) juxtaposed between the apposed male and female pronuclei (blue) and nucleating astral microtubules (green; (G) inset, total tubulin [red] and DNA [blue]; incorporated sperm axoneme [small arrows]). (C,H) fixed metaphase spindles immunostained for microtubules (green) and DNA (blue), showing eccentric and tangentially-oriented IVF spindles, are distinct from radially oriented, cortically distal ICSI spindles (H: sperm axoneme, small arrows). White bars: the orthogonal anaphase chromosome separation plane. (D,I) A fixed, immunostained IVF anaphase spindle with enhanced spindle microtubules (D: green) signaling cortical cleavage furrow initiation (arrow) compared to an ICSI anaphase spindle lacking cortical microtubule interaction at the furrow (I: green, arrow). Both cleavage planes are orthogonal to the separating sister chromosomes (blue). (E,J) Equal IVF telophase (E) cleavage versus unequal ICSI telophase cleavage $(\mathbf{J})$ despite asymmetric cleavage furrows (arrows) progressing orthogonally to midbodies (green, arrowheads; blue, DNA; small arrowhead: centrosome; small arrows: sperm axoneme). (K1-K4, L1-L4). TLVM images of two ICSI rhesus zygotes, both with eccentric, radially aligned, apposed male and female pronuclei $(\mathrm{K} 1, \mathbf{L 1})$. The cleavage furrow begins near the previously intact MPn (K2, L2: arrows), progressing asymmetrically (K3, L3: arrows) but not through the second polar bodies $(\mathrm{Pb})$. (K4,L4) anti-tubulin (green) and Hoechst DNA (blue) immunostaining of (K3,L3) zygotes fixed 2 min post-live imaging. Central microtubule midbodies (green, arrowheads) lie orthogonal to the progressing cleavage furrows (arrows). Polar bodies are excluded from the cleavage planes (K4: $\mathrm{Pb}$; L4: * marks lost polar bodies). HMC images: (A,F,K1-K3,L1-L3); B: triple-labeled for microtubules (green), $\gamma$-tubulin (red), and DNA (blue); (G) quadruple-labeled for glutamylated-microtubules/ $\gamma$-tubulin (green), total tubulin (red), and DNA (blue); (K4,L4) microtubules (green) and DNA (blue). Pb: polar bodies. Bars $=20 \mu \mathrm{m}$.

Microtubule and DNA patterns in mitotic parthenogenotes are presented in Supplemental Fig. S6. Identical microtubule and DNA configurations were observed regardless of the artificial activation methodology employed. Cytoplasmic microtubule patterns in late interphase parthenogenotes were random in the absence of sperm centrioles. At mitosis, a bipolar anastral central cytoplasmic spindle assembled with aligned female chromosomes, probably directed by specific microtubule-based molecular motor proteins that participate in non-centrosomal 
spindle assembly ${ }^{59}$. By anaphase, spindle microtubules were sparse in the equatorial region through which cleavage furrow progression would ensue, and despite asymmetrical cleavage furrow formation and progression, no spindle microtubule:cortex interactions were observed $(n=6)$.

Collectively, a strong cortical attraction of the MPn to the zygote's oolemma directs cleavage furrow formation as mitosis ensues. Absent any paternal contribution, female pronuclei lack dominant MTOCs (centrioles) to direct motility events within the activated cytoplasm, including mitotic spindle assembly. Parthenogenotes demonstrate lower affinity for cortical pronuclear positioning, central cytoplasmic mitotic spindles with enormous spindle pole microtubule assembly, but no anaphase microtubule:cortex interactions despite asymmetric furrows that pass orthogonally to the chromosome separation plane. The molecular signals required to initiate cleavage furrow assembly and progression in parthenogenotes are currently not known.

\section{Discussion}

Notwithstanding excellent investigations conducted on the best-studied mammalian model, the mouse, crucial information is scarce regarding early fertilization events influencing axis formation in non-rodent mammals ${ }^{30,60-62}$. Controversy surrounds the evidence indicating that developmental information encoded within oocytes and early zygotes determines axis development of the blastocyst; strengths and limitations of the various experimental designs have been discussed ${ }^{17}$. For ART clinics, whether understanding of early morphological characteristics in the human zygote can translate to selection of the highest quality human embryos is enormously important ${ }^{36}$. Animal models like NHPs provide more translatable relevance to humans ${ }^{13,63}$. For example, atypical early fertilization events after ICSI, such as abnormal nuclear remodeling, asynchronous apical sperm chromatin decondensation, and inappropriate retention of sperm proteins during interphase, have been identified in NHP zygotes $^{64,65}$, which may have important implications for ART, considering the growing use of ICSI for oocyte insemination.

Here, observational information in macaques and baboons, using continuous live multidimensional (z-stack) TLVM recordings, from sperm incorporation though first division, is provided, a crucial improvement over single-plane imaging or only fixed-stage microscopic examinations. Multidimensional imaging permits observing independent male and female pronuclear behaviors, pronuclear migration rates and ultimate oolemma orientation and cortical marking sites (i.e., polar bodies and sperm entrance sites) as well as cleavage furrow initiation, progression, and cytokinesis in $4 \mathrm{D}(3 \mathrm{D}+$ time $)$. NHP oocytes and zygotes, as large ovoid spheres, benefit from $4 \mathrm{D}$ imaging, where detailed motility analysis on the entire fertilization process can be studied in detail to identify robust embryos. Complementing multidimensional TLVM analysis was the fixation of mitotic zygotes in the investigation of spindle arrangements and cleavage events as influenced by pronuclear orientation, including analysis of the same zygote undergoing TLVM imaging within minutes of the last captured frame (Fig. 5). Additionally, manipulating the genetic constitution of zygotes by forming parthenogenotes and androgenotes is informative regarding the interactions of male and female pronuclei within the activated zygote's cytoplasm. These observational studies lay a foundation for studying the underlying molecular mechanisms driving asymmetric pronuclear apposition, spindle assembly, and cleavage initiation in a higher-order primate model with better potential extrapolations to humans than studies in mice.

This study identified distinct differences in sperm head behavior between NHP IVF- versus ICSI-inseminated zygotes (Figs 1 and 2). IVF sperm appeared tightly bound to the oolemma following membrane penetration, with sperm head movement mediated by increased bulk membrane flow during second meiosis completion (Fig. 1; Suppl Video 1). Typically, IVF sperm heads decondensed and remained closely associated with the cortical penetration site (Suppl Video 2). In contrast, ICSI fertilization showed different dynamics following sperm microinjection (Fig. 2). Placement of the immobilized sperm into the oocyte's cortex induced sperm head displacement along the inner plasma membrane, probably from cytoplasmic streaming forces (Suppl Video 3). Rarely did cortical ICSI sperm remain at the microinjection site but always showed a strong cortical retention.

The significance of these observations relates to the eccentric orientation of the male and female pronuclei after sperm head decondensation, pronuclear assembly, and migration (Table 1). NHP zygotes have highly eccentric, cortically positioned, juxtaposed male and female pronuclei post apposition, with IVF zygotes showing radial pronuclei orientations relative to the overlying adjacent cortex while ICSI zygotes vary their pronuclear alignment between radial and para-tangential alignments (Table 1; Figs 1 and 2). Furthermore, TLVM analysis showed many ICSI zygotes with atypical pronuclear cytoplasm movements after apposition, including pronuclear tumbling, separation/ejection, and slow drifting away from the cortex prior to mitosis, reinforcing how the lack of sperm:oolemma binding might affect the final ICSI pronuclear cytoplasm positioning that influences cleavage initiation and progression.

Interestingly, while NHP zygotes closely mimic the early fertilization events observed in human zygotes ${ }^{63}$, NHP pronuclear positioning post-apposition is not equivalent to humans, that show more central cytoplasmic apposition post female pronuclear migration ${ }^{37-49}$, like that observed in the mouse ${ }^{57}$. Past studies in human zygotes have shown that pronuclear morphology (nucleoli) and, perhaps, orientation to the polar body impact embryo quality, blastocyst development, and pregnancy establishment, irrespective of fertilization method ${ }^{41,42}$. A recent TLVM analysis of human ICSI zygotes, however, reported nearly half to have cortically or intermediately positioned cytoplasmic apposed male and female pronucle ${ }^{41}$. It would be interesting to know how human ICSI pronuclear cytoplasmic position and alignment influences cleavage events and translates to human pregnancy outcomes. Taken together, these data indicate how early NHP fertilization events vary between conventional IVF and ICSI insemination, with potentially important implications for clinical ART outcomes.

Neither NHP IVF or ICSI sperm induced a post-insemination incorporation cone (Figs 1 and 2; Suppl Videos 1 and 3), a structure in mouse oocytes that supports first cleavage plane assembly by preferentially aligning the mitotic spindle along the $\mathrm{SEP}^{26,33}$. Interestingly, human zygotes reportedly assemble transient insemination cones a few hours after IVF or ICSI insemination, although the frequency of occurrence appears very low ${ }^{40,41}$, 
while in mice, the site of sperm entry and the $2^{\text {nd }} \mathrm{PB}$ axis appear to be important determinants for axial development of the mouse blastocyst ${ }^{28,29}$.

A previous study on ICSI-fertilized rhesus oocytes tagged at the SEP with a fluorescent bead suggested a strong influence of the NHP SEP on early axis specification; this was based on observing embryos at the 2-cell and blastocyst stages after in vitro culture ${ }^{30}$. However, continuous multidimensional TLVM in baboon zygotes showed most fluorescent beads tagging the MIP ended up within the furrow region at the end of cytokinesis, resulting from bulk membrane flow directed toward the first division plane as opposed to either cleavage initiation or progression through this site (Fig. 4; Table 3; Suppl Video 6). Attempts to trace where the MIP site resides in the expanded baboon blastocysts grown in vitro was confounded by multidimensional TLVM observations showing bead detachments/reattachments during preimplantation development, with overall in vitro development rates to the blastocyst stage at $27 \%$ (3/11]; Fig. 4). While the MIP did not appear to influence cleavage plane initiation or progression, cleavage furrows overwhelmingly began at the cortical site near where the previously intact MPn resided and progressing meridionally through the animal pole $\left(2^{\text {nd }} \mathrm{PB}\right)$, regardless of whether the ICSI apposed pronuclei were oriented radially or para-tangentially to the overlying cortex (Fig. 3; Table 2). In this regard, Hiiragi and Solter' ${ }^{34}$ observation that the mouse cleavage plane was anticipated by the plane of the approaching male and female pronuclei appears to explain NHP cleavage initiation also. Tracing a line along the long axis of the NHP apposed pronuclei appears to be an accurate predictor of cleavage plane progression.

For non-rodent mammals, the influence of the animal pole and the SEP shows varying influence on division planes. Human IVF zygotes assemble central cytoplasm mitotic spindles with furrow invagination beginning equally from opposite cortical regions and passing close to the $2^{\text {nd }} \mathrm{PB}$ and through the SEP, perhaps indicating a role for the SEP in human axis specification ${ }^{38,41}$, while the SEP in IVF sheep zygotes does not appear to influence the first cleavage plane ${ }^{60}$.

Mouse oocyte shape changes, induced either artificially by external mechanical force or by the sperm incorporation cone at insemination, influence the first cleavage plane assembly by aligning the spindle to the $\mathrm{SEP}^{26,33}$. In the early NHP ICSI zygote, multidimensional TLVM showed significant cortical contraction/expansion cycles, sometimes visualized as pulsating images on the TLVM recordings. The appearance of contractions coincided with microtubule aster assembly from the base of the incorporated sperm, arising from sperm astral microtubule interaction with the cortical membrane. Cytoplasmic expansion generally occurred as mitosis ensued, with a net outflow of cytoplasmic constituents away from the assembling first mitotic spindle.

Our TLVM recordings also occasionally detected a "cytoplasmic wave" emanating from the growing sperm aster after ICSI, as described during early human fertilization ${ }^{41}$. However, NHP cleavage plane assembly and progression appears to be more related to the actual male pronuclear site, pronuclear orientation, and the inherited sperm centrosome cytoplasmic positioning by the end of interphase. While both IVF and ICSI NHP sperm contribute centrioles that assemble the zygotic centrosome that nucleates sperm astral microtubules for female pronuclear migration ${ }^{52}$, no statistical differences between IVF and ICSI zygotes' female pronuclear migration rates were identified (Table 1), although less robust ICSI sperm:cortical interaction seemed to shorten migration distances between distal pronuclei. The NHP reconstituted centrosome, after fertilization, duplicates during interphase $\mathrm{e}^{14}$, lying between the opposing pronuclear membranes (Fig. $5^{52}$ ) and defining the first mitotic spindle poles as they do for most mammals except mice ${ }^{12}$. Mice do not assemble a microtubule-based sperm aster with pronuclear movements directed by cortical interphase microtubule arrays and microfilaments ${ }^{12,66-69}$, and the maternal centrosomes that coalesce about the nuclear surfaces of concentric male and female pronuclei prior to mitosis direct spindle assembly (Supplemental Fig. S2 ${ }^{68}$ ). However, how pronuclear orientation differed between NHP IVF and ICSI zygotes at the end of interphase appeared to markedly influence eccentric mitotic spindle assembly and cytoplasmic orientation (Fig. 5).

In IVF zygotes, radially oriented apposed male and female pronuclei, with the MPn closest to the overlying adjacent cortex (Fig. 1; Table 1), favors assembling eccentric para-tangential bipolar mitotic spindles, which initiate division planes that move across the greater diameter of the zygote, forming two cells with equal daughter blastomeres (Fig. 5; Suppl Fig. S3). Conversely, ICSI mitotic zygotes show para-tangential pronuclear orientations that have centrosome positions shifted $90^{\circ}$ relative to IVF mitotic spindles (Fig. 5). At anaphase, ICSI spindles show reduced spindle microtubule:cortex interactions (compare Fig. 5D,I,K4,L4; 14\%), which might critically impact not only furrow initiation signaling but mitotic spindle stabilization during division, so that the rapidly invaginating furrow does not sweep aside the spindle apparatus during cytokinesis to form abnormal binucleate daughters. Mitotic spindle re-orientation within the cytoplasm may be impacted by cortical streaming forces acting on the less well-anchored ICSI spindle, which could account for the greater incidence of unequal sized daughter blastomeres observed post-cytokinesis (Table 2).

The significance of the MPn in cleavage initiation was reinforced by our observations on (i) placement of the microinjected sperm into the central cytoplasm of the oocyte; (ii) cytoplasmic pronuclear location and cleavage in parthenogenetic zygotes; and (iii) pronuclear location and cleavage in androgenetic oocytes. For sperm central cytoplasmic deposit, the female migrated to the male quickly (Table 1) but once apposed, moved precipitately toward the cortex in tandem (Fig. 2), with cleavage beginning at the cortex closest to the previously intact MPn, usually identified by the attached incorporated sperm axoneme. In parthenogenotes, formed by either chemical activation (ionomycin/6-DMAP) or by microinjection of a crude sperm extract, the female pronuclei generally moved toward the central cytoplasm, away from the cortex (Supplemental Fig. S4) and assembled asymmetric cleavage furrows, mostly from the polar body region, but sometimes, from random cortical sites (Table 2; Supplemental Fig. S5).

Investigations on fixed mitotic parthenogenotes showed the random cortical microtubule arrays formed after oocyte activation in the absence of male-contributed centrioles as well as cytoplasmic centric anastral, acentriolar bipolar spindles at metaphase (Supplemental Fig. S6). Interestingly, mitotic chromosome separation at anaphase was concomitant with large spindle pole microtubule assembly that appeared to be excluded from any cortical 
region. Cleavage progression, however, was orthogonal to the separating chromosomes. These NHP parthenogenote observations support findings in mouse parthenogenotes, where, in the absence of an SEP, cleavage planes were observed to be random ${ }^{70}$.

Lastly, we investigated pronuclear events and cleavage initiation in androgenotes produced either by meiotic spindle enucleation followed by ICSI or removal of the FPn shortly after ICSI and FPn formation. Regardless of genetic constitution, enucleated oocytes had male pronuclei with a strong cortical attraction (Supplemental Fig. S4) and cleavage initiation at the cortex closest to the previously intact MPn as observed for ICSI-fertilized zygotes (Supplemental Fig. S5). We speculate that sperm centrosomal microtubules, interacting with unknown cortical proteins, drive male pronuclear cortical positioning, although the exact mechanism remains unknown. Collectively, these observations show a strong attraction of the paternal pronucleus for the cortex and eccentric cleavage initiation, which may reflect the assembly of the sperm microtubule aster from the reconstituted centrosomes following insemination.

In summary, NHP zygotes display features distinct from more traditionally studied rodent models, with fundamental differences in pronuclear cytoplasmic positioning/orientation, spindle assembly in relation to inherited centrosome placement, and cleavage initiation/progression. ICSI cleavage initiation and progression is independent of a SEP but favors division through the animal pole. Nonhuman primates follow the pattern of paternal inheritance of centrosomes for directing motility events during fertilization seen also in humans, but not mice ${ }^{12}$. Differences between NHP and mouse zygotes are profound in many aspects of the fertilization process, including sperm incorporation mechanics, paternal centriole inheritance, male and female pronuclear apposition methods, atypical cytoplasmic position of juxtaposed pronuclei, mitotic bipolar spindle assembly mechanisms, and cytokinesis characteristics, among others (Supplemental Table 1). Identifying these unique characteristics has helped to define how NHP zygotes accomplish zygotic division as the first step in the process of embryonic axis formation and sets the stage for investigation of the molecular signals directing these critical events.

\section{Methods}

Ethics statement. All research protocols complied with the National Institute of Health's Office of Laboratory Animal Welfare Guide for the Care and Use of Laboratory Animals regulations under the full approval of protocols by the Institutional Animal Care and Use Committees (IACUCs) of Oregon National Primate Research Center (NOI \#s 0483; 0095), Magee-Womens Research Institute \& Foundation at the University of Pittsburgh (IACUC \#s 0802725, 0804730), and the Texas Biomedical Research Institute in San Antonio Texas (formerly known as the Southwest Foundation for Biomedical Research; IACUC\#s 1235 MF, 1168 MF, 986 PC 21 , and $1235 \mathrm{MF}$ ).

Non-human primate gamete collections. Ovarian stimulation of female rhesus monkeys exhibiting regular menstrual cycles was induced with exogenous gonadotrophin administration as previously described ${ }^{71}$. Follicular aspirations were performed approximately $27-30 \mathrm{~h}$ post-hCG via laparoscopy for macaques (rhesus, cynomolgus) and around $32-36 \mathrm{~h}$ post-hCG for the baboon ${ }^{52,71}$. Oocytes at metaphase II-arrest, exhibiting expanded cumulus cells, a distinct perivitelline space, and first polar body, were selected for IVF fertilization, artificial activation, or ICSI ${ }^{55,64,72}$.

Fertilization, artificial activation, and preparing androgenetic NHP oocytes. Rhesus and cynomolgus sperm were collected by penile band electroejaculation and fertilized in vitro as reported ${ }^{73}$. Baboon sperm was collected by rectal probe electroejaculation under approved protocols ${ }^{71}$. ICSI fertilization using $6-7 \mu \mathrm{m}$ microinjection needles (Humagen, Inc., Charlottesville, VA) was accomplished by the methods of Hewitson et al. ${ }^{55}$. In some cases, a small $(\sim 3 \mu \mathrm{m})$ fluorescent bead (Polysciences, Inc, Warrington, PA) coated with $350 \mu \mathrm{g} / \mathrm{ml} \mathrm{phy}-$ tohemagglutinin (PHA; Sigma, St. Louis, MO) was placed onto the membrane to mark the ICSI site after sperm delivery, as described by Piotrowska and Zernicka-Goetz $\mathrm{Z}^{70}$. Artificial activation (parthenogenesis) was accomplished by either activation with $5 \mu \mathrm{m}$ ionomycin/ $1.9 \mathrm{mM}$ 6-dimethylaminopyridine (6-DMAP $)^{74}$ or injection of an NHP crude sperm extract (120-240 $\left.\mathrm{pg} \mathrm{ml}^{-175}\right)$. Androgenote production was accomplished by ICSI of oocytes after enucleation of the second meiotic spindle or the female pronucleus $4-6$ hrs post ICSI using a $22-\mu \mathrm{m}$ pipet as previously reported ${ }^{76,77}$.

Immunocytochemistry. Selected zygotes were fixed either after zona pellucida removal overnight in $2 \%$ formaldehyde or for $15 \mathrm{mins}$ in absolute methanol $\left(-20^{\circ} \mathrm{C}\right)$ following extraction in a detergent based stabilization buffer $^{78}$. Primary antibodies $\left(1 \mathrm{hr} ; 37^{\circ} \mathrm{C}\right)$ included anti-tubulin $(\mathrm{E}-7 ; 1: 20$; Developmental Studies Hybridoma Bank, Iowa City, IA), $\gamma$-tubulin (1:500; gift of Dr. T. Stearns, Stanford University), myosin II (gift of Dr. P de Lanerolle, University of Illinois-Chicago); pericentrin (PCM; gift of Dr. S. Doxsey, University of Massachusetts Medical School); and detyrosinated anti-tubulin (glu-MTs; 1:500; Millipore, Temecula, CA). Primary antibodies were detected with appropriate fluorescein- or rhodamine-labeled secondary antibodies (1:200; Molecular Probes, Eugene, OR.). DNA was labeled with $10 \mu \mathrm{g} / \mathrm{ml}$ Hoechst 33345 (Sigma Chemical Corporation, St Louis, MO). Images were taken by conventional epifluorescence or laser-scanning confocal microscopy (Leica LCS, Leica Microsystems Heidelberg, Germany).

Time-lapse video microscopy. (TLVM) was performed on an inverted Nikon TE-2000U using Hoffman Modulation Contrast (HMC) optics (x 20). An environmental chamber equipped with a heater to maintained $37^{\circ} \mathrm{C}$ encased the microscope (Fryer Environmental Control Chamber, Chicago, IL). A $35 \mathrm{~mm}$ glass bottom Petri dish (MatTek, Ashland MA) was placed into a Tokai Stage Top Chamber Unit (Model: INU-ONI-F1; Tokai Hit, Japan) mounted to the microscope stage to maintain $\mathrm{pH}$ by a constant flow of $5 \% \mathrm{CO}_{2}$ gas. Images were collected using an ORCA-ER digital camera (Hamamatsu Photonics, Inc., Japan) using Metamorph software (Molecular 
Devices, Sunnyvale, CA). Z-series control for acquisition of image stacks was accomplished with a $\mathrm{z}$-motor (Model MFC-2000; Applied Scientific Instruments, Eugene, OR) and controlled by Metamorph. Z-series images $(5 \mu \mathrm{m})$ of pronuclear development, apposition and migration were collected every $5-15$ mins while mitosis was collected every $2 \mathrm{~min}$.

Imaging and analysis. Conventional fluorescence microscopy was performed using a Nikon Eclipse E1000 microscope with high numerical aperture objectives and data recorded digitally using a cooled CCD camera (Hamamatsu Instruments Inc., Japan) and Metamorph software (Universal Imaging, West Chester, PA). Laser-scanning confocal microscopy utilized a Leica SP-2 equipped with argon and helium-neon lasers. Selected image panels from TLVM recordings were prepared with time listed in hrs:min. For all images, final panels for plates were prepared from selected tagged imaged file format (tiff) images in Adobe Photoshop (Adobe Systems, Inc, San Jose, CA).

Statistics. Means \pm standard error (s.e.) were determined by an online tool at EasyCalculations.com. Statistical significance was determined with the Student's $t$ test (two-tailed), with actual $p$ valves expressed, and was performed with GraphPad Software, Incorporated (La Jolla, CA). Significance was determined if $\mathrm{p}<0.05$.

\section{Data availability}

Datasets generated and/or analyzed during this study are available from the corresponding author on reasonable request as set forth in the journal guidelines.

Received: 21 June 2019; Accepted: 3 October 2019;

Published online: 25 October 2019

\section{References}

1. Boulet, S. L. et al. Trends in use of and reproductive outcomes associated with intracytoplasmic sperm injection. JAMA. 313, 255-63 (2015).

2. Peultier, A. S., Fréour, T., Cazenave, N. \& Barrière, P. Fertilization failure in IVF and ICSI. J. Gynecol. Obstet. Bio. Reprod. (Paris) 44, 380-386 (2015)

3. Grimstad, F. W. et al. Use of ICSI in IVF cycles in women with tubal ligation does not improve pregnancy or live birth rates. Hum. Reprod. 31, 2750-2755 (2016).

4. Schwarze, J. E. et al. Is there a reason to perform ICSI in the absence of male factor? Lessons from the Latin American Registry of ART. Hum. Reprod. Open (2):hox013. Published 2017 Aug 30, https://doi.org/10.1093/hropen/hox013 (2017).

5. Dang, V. Q. et al. The effectiveness of ICSI versus conventional IVF in couples with non-male factor infertility: study protocol for a randomized controlled trial. Hum. Reprod. Open, https://doi.org/10.1093/hropen/hoz006 (2019).

6. Koedooder, R. et al. The vaginal microbiome as a predictor for outcome of in vitro fertilization with or without intracytoplasmic sperm injection: a prospective study. Hum. Reprod. pii: dez065, https://doi.org/10.1093/humrep/dez065 (2019).

7. Clift, D. \& Schuh, M. Restarting life: fertilization and the transition from meiosis to mitosis. Nat. Rev. 14, 549-562 (2013)

8. Rossant, J. Making the Mouse Blastocyst: Past, Present, and Future. Curr. Top Dev. Biol. 117, 275-88, https://doi.org/10.1016/ bs.ctdb.2015.11.015 (2016).

9. Leung, C. Y., Zhu, M. \& Zernicka-Goetz, M. Polarity in Cell-Fate Acquisition in the Early Mouse Embryo. Curr. Top Dev. Biol. 120, 203-34, https://doi.org/10.1016/bs.ctdb.2016.04.008. (2016).

10. White, M. D., Zenker, J., Bissiere, S. \& Plachta, N. Instructions for Assembling the Early Mammalian Embryo. Develop. Cell 45, 667-679 (2018).

11. Martínez-Frías, M. L. Assessing pre-implantation embryo development in mice provides a rationale for understanding potential adverse effects of ART and PGD procedures. Am. J. Med. Genet. Part A 158A, 2526-2533 (2012).

12. Schatten, G. The centrosome and its mode of inheritance: the reduction of the centrosome during gametogenesis and its restoration during fertilization. Dev. Biol. 165, 299-335 (1994).

13. Simerly, C. \& Schatten, G. Utility of animal models for human embryo culture: nonhuman primates in embryo culture: advances, controversies, and future considerations (eds Pool, T., Smith, Gary \& Swain, J. E.). Methods Mol. Biol. 912, 39-59 (2012).

14. Fishman, E. L. et al. A novel atypical sperm centriole is functional during human fertilization. Nat. Commun. 9(1), 2210, https://doi. org/10.1038/s41467-04678-8 (2018).

15. Avidor-Reiss, T. \& Fishman, E. L. It takes two (centrioles) to tango. Reprod. pii:REP-18-0350.R1, 10.150/REP-18-0350 (2018).

16. Else, H. Genomics institute to close world-leading animal facility. Nature 569, 612 (2019).

17. Johnson, M. H. From mouse egg to mouse embryo: polarities, axes, and tissues. Annu. Rev. Cell. Dev. Biol. 25, 483-512 (2009)

18. Chen, Q., Shi, J., Tao, Y. \& Zernicka-Goetz, M. Tracing the origin of heterogeneity and symmetry breaking in the early mammalian embryo. Nat. Comm. 9, 1-11 (2018).

19. Ajduk, A. \& Zernicka-Goetz, M. Quality control of embryo development. Mol. Asp. Med. 34, 903-918 (2013).

20. Zernicka-Goetz, M. The first cell-fate decisions in the mouse embryo: destiny is a matter of both chance and choice. Curr. Op. Genet. Develop. 16, 406-412 (2006)

21. Zernicka-Goetz, M. Cleavage pattern and emerging asymmetry of the mouse embryo. Nature Rev. 6, 919-928 (2005).

22. Rossant, J. \& Tam, P. P. L. Emerging asymmetry and embryonic patterning in early mouse development. Devel. Cell 7, 155-164 (2004).

23. Gardner, R. L. The early blastocyst is bilaterally symmetrical, and its axis of symmetry is aligned with the animal-vegetal axis of the zygote in the mouse. Development 124, 289-301 (1997).

24. Gardner, R. L. The axis of polarity of the mouse blastocyst is specified before blastulation and independently of the zona pellucida. Hum. Reprod. 22, 798-806 (2006).

25. Plusa, B., Grabarek, J. B., Piotrowska, K., Glover, D. M. \& Zernicka-Goetz, M. Site of the previous meiotic division defines cleavage orientation in the mouse embryo. Nature Cell Biol. 4, 811-815 (2002a).

26. Plusa, B. et al. The first cleavage of the mouse zygote predicts the blastocyst axis. Nature 434, 392-395 (2005).

27. Hiiragi, T. \& Solter, D. Mechanism of first cleavage specification in the mouse egg. Cell Cycle 4(5), 661-664 (2005).

28. Piotrowska, K. \& Zernicka-Goetz, M. Role for sperm in spatial patterning of the early mouse embryo. Nature 409, 517-521 (2001).

29. Plusa, B., Piotrowska, K. \& Zernicka-Goetz, M. Sperm entry position provides a surface marker for the first cleavage planes of the mouse zygote. Genesis 32, 193-198 (2002b).

30. Piotrowska-Nitsche, K., Yang, S. H., Banta, H. \& Chan, A. W. Assisted fertilization and embryonic axis formation in higher primates. Reprod. Biomed. Online 18, 382-390 (2009). 
31. Piotrowska-Nitsche, K. \& Chan, A. W. S. Effect of sperm entry on blastocyst development after in vitro fertilization and intracytoplasmic sperm injection-mouse model. J. Assist. Reprod. Genet. 30, 81-89 (2013).

32. Kurotaki, Y., Hatta, K., Najao, K., Nabeshima, Y. \& Fujimori, T. Blastocyst axis is specified independently of the early cell lineage but aligns with the ZP shape. Science 316, 719-723 (2007).

33. Gray, D. et al. First cleavage of the mouse embryos responds to change in egg shape at fertilization. Curr. Biol. 14, 397-405 (2004).

34. Hiiragi, T. \& Solter, D. First cleavage plane of the mouse egg is not predetermined but defined by the topology of the two apposing pronuclei. Nature 430, 360-364 (2004).

35. Hiiragi, T. et al. Where do we stand now? Mouse early embryo patterning meeting in Freiburg, Germany (2005). Int. J. Dev. Biol. 50, 581-588 (2006)

36. Gardner, D. K. \& Balaban, B. Assessment of human embryo development using morphological criteria in an era of time-lapse, algorithms, and 'OMICS': is looking good still important? Mol. Hu. Reprod. 22(10), 704-718 (2016).

37. Payne, D., Flaherty, S. P., Barry, M. F. \& Mathews, C. D. Preliminary observations on polar body extrusion and pronuclear formation in human oocytes using time-lapse video cinematography. Hum. Reprod. 12, 532-541 (1997).

38. Mio, Y. \& Maeda, K. Time-lapse cinematography of dynamic changes occurring during in vitro development of human embryos. Am. J. Obstet. Gynecol. 199, 660.e1-660.e5 (2008).

39. Aguilar, J. et al. The human first cell cycle: impact on implantation. Reprod. BioMed. Online 28, 475-484 (2014).

40. Iwata, K. \& Mio, Y. Observation of human embryonic behavior in vitro by high-resolution time-lapse cinematography. Reprod. Med. Biol. 15, 145-154 (2016).

41. Coticchio, G. et al. Focused time-lapse analysis reveals novel aspects of human fertilization and suggests new parameters of embryo viability. Hum. Reprod. 33(1), 23-31 (2018).

42. Scott, L., Alvero, R., Leondires, M. \& Miller, B. The morphology of human pronuclear embryos is positively related to blastocyst development and implantation. Hum. Reprod 15, 2394-2403 (2000).

43. Giannaroli, L. et al. Pronuclear morphology and chromosomal abnormalities as scoring criteria for embryo selection. Fert. Steril. 80 , 341-349 (2003)

44. Giannaroli, L. et al. Oocyte euploidy, pronuclear zygote morphology and embryo chromosomal complement. Hum. Reprod. 22, 241-249 (2007)

45. Kattera, S. \& Chen, C. Developmental potential of human pronuclear zygotes in relation to their pronuclear orientation. Hum. Reprod. 19(2), 294-299 (2004).

46. Tesarik, J. \& Greco, E. The probability of abnormal preimplantation development can be predicted by a single static observation on pronuclear stage morphology. Hum. Reprod. 14, 1318-1323 (1999).

47. Chen, C. \& Kattera, S. Comparison of pronuclear zygote morphology and early cleavage status of zygotes as additional criteria in the selection of day 3 embryos: a randomized study. Fert. Steril. 85(2), 347-352 (2006).

48. Garello, C. et al. Pronuclear orientation, polar body placement, and embryo quality after intracytoplasmic sperm injection and invitro fertilization: further evidence for polarity in human oocytes? Hum. Reprod. 14, 2588-2595 (1999).

49. Kai, Y., Iwata, K., Iba, Y. \& Mio, Y. Diagnosis of abnormal human fertilization status based on pronuclear origin and/or centrosome number. J. Assist. Reprod. Genet. 32, 1589-1595 (2015).

50. Desai, N. \& Gill, P. Blastomere cleavage plane orientation and the tetrahedral formation are associated with increased probability of a good-quality blastocyst for cryopreservation or transfer: a time-lapse study. Fert. Steril. pii.S0015-0282(19)30130-X, https://doi. org/10.1016/j.fertnstert.2019.12.019 (2019).

51. Palermo, G. D. et al. Intracytoplasmic sperm injection: state of the art in humans. Repro. 154(6), F93-F110 (2017).

52. Wu, G.-J., Simerly, C., Zoran, S. S., Funte, L. R. \& Schatten, G. Microtubule and chromatin dynamics during fertilization and early development in rhesus monkeys and regulation by intracellular calcium ions. Biol. Reprod. 55, 260-270 (1996).

53. Simerly, C. \& Navara, C. S. The Sperm Centriole: Its effect on the developing embryo. In: Human Preimplantation Embryo Selection (ed. Cohen, J. \& Elder, K.) Taylor \& Francis Group, Boca Raton, Florida (2007).

54. Niwayama, R. et al. Bayesian inference of forces causing cytoplasmic streaming in Caenorhabditis elegans embryos and mouse oocytes. Plos One 11(7), e0159917, https://doi.org/10.1371/journal.pone.0159917 (2016).

55. Hewitson, L., Takahashi, D., Dominko, T., Simerly, C. \& Schatten, G. Fertilization and embryo development to blastocysts after intracytoplasmic sperm injection in the rhesus monkey. Hum. Reprod. 13, 3449-3455 (1998).

56. Johnson, M. H. So, what exactly is the role of the spermatozoon in first cleavage? Reprod. BioMed. Online 6(2), 163-167 (2003).

57. Gardner, R. L. \& Davies, T. J. An investigation of the origin and significance of bilateral symmetry of the pronuclear zygote in the mouse. Hu. Reprod. 21(2), 492-502 (2006).

58. Simerly, C., Nowak, G., de Lanerolle, P. \& Schatten, G. Differential expression and functions of cortical myosin IIA and IIB isotypes during meiotic maturation, fertilization, and mitosis in mouse oocytes and embryos. Mol Biol Cell. 9, 2509-2525 (1998).

59. Mountain, V. et al. The kinesin-related protein, HSET, opposes the activity of Eg5 and cross links microtubules in the mammalian mitotic spindle. J. Cell. Bio. 147(2), 351-365 (1999).

60. Hosseini, S.-M. et al. The principal forces of oocyte polarity are evolutionary conserved but may not affect the contribution of the first two blastomeres to the blastocyst development in mammals. Plos ONE 11(3), 0148382, https://doi.org/10.1371/journal. pone.0148382 (2016)

61. Park, S. K., Won, C., Choi, Y.-J., Kang, H. \& Roh, S. The leading blastomere of the 2-cell stage parthenogenetic porcine embryo contributes to the abembryonic part first. J. Vet. Med. Sci. 7, 569-576 (2009).

62. Kim, K., Park, S. \& Roh, S. Lipid-rich blastomeres in the two-cell stage of porcine parthenogenotes show bias toward contributing to the embryonic part. Am. Reprod. Sci. 130, 91-98 (2012).

63. Hewitson, L. \& Schatten, G. The use of primates as models for assisted reproduction. Reprod. Biomed. Online 5, 50-55 (2002).

64. Hewitson, L. et al. Births of ICSI monkeys: Unique checkpoints during the first cell cycle of fertilization. Nat. Medicine 5(4), 431-433 (1999).

65. Ramalho-Santos, J. et al. ICSI choreography: Fate of sperm structures after monospermic ICSI and first cell cycle implications. Hum. Reprod. 15, 2610-2620 (2000).

66. Maro, B. Fertilization and the cytoskeleton in the mouse. Bioessays 3(1), 18-21 (1985)

67. Schatten, G., Simerly, C. \& Schatten, H. Microtubules in mouse oocytes, zygotes, and embryos during fertilization and early development: unusual configurations and arrest of mammalian fertilization with microtubule inhibitors. Ann. N. Y. Acad. Sci. 466, 945-948 (1985).

68. Schatten, H., Schatten, G., Mazia, D., Balczon, R. \& Simerly, C. Behavior of centrosomes during fertilization and cell division in mouse oocytes and in sea urchin eggs. Proc. Natl. Acad. Sci. USA 83, 105-109 (1986a).

69. Schatten, G. et al. Latrunculin inhibits the microfilament-mediated processes during fertilization, cleavage and early development in sea urchins and mice. Exp. Cell Res. 166, 191-208 (1986b).

70. Piotrowska, K. \& Zernicka-Goetz, M. Early patterning of the mouse embryo-contributions of sperm and egg. Develop. 129, 5803-5858 (2002).

71. Simerly, C. R. et al. Assisted reproductive technologies with baboons generate live offspring: a nonhuman primate model for ART and reproductive sciences. Repro. Sci. J. 17, 917-930 (2010).

72. Simerly, C. et al. Embryogenesis and blastocyst development after somatic cell nuclear transfer in nonhuman primates: overcoming defects caused by meiotic spindle extraction. Dev. Biol. 276, 237-252 (2004). 
73. Bavister, B., Boatman, D. E., Leibfried, L., Loose, M. \& Vernon, M. W. Fertilization and cleavage of rhesus monkey oocytes in vitro. Biol. Reprod. 28, 983-99 (1983).

74. Navara, C., First, N. \& Schatten, G. Microtubule organization in the cow during fertilization, polyspermy, parthenogenesis and nuclear transfer. Dev. Biol. 162, 29-40 (1994).

75. Swann, K. \& Lai, F. A. A novel signaling mechanism for generating $\mathrm{Ca}^{2+}$ oscillations at fertilization in mammals. Bioessays 19(5), 371-378 (1997).

76. Simerly, C. et al. Molecular correlates of primate nuclear transfer failures. Science 300, 297 (2003).

77. Simerly, C. et al. Embryogenesis and blastocyst development after somatic cell nuclear transfer in non-human primates [nhp-scnt]: overcoming defects caused by meiotic spindle extraction. Dev. Biol. 276, 237-252 (2004).

78. Simerly, C. \& Schatten, G. Techniques for Localization of Specific Molecules in Oocytes and Embryos. Meth. Enzymol. 225, 516-552 (1993).

\section{Acknowledgements}

This study is dedicated to the memory of Mr. Kevin Grund, a friend and dedicated nonhuman primate research colleague, whose unfailing devotion to advancing scientific study was invaluable to our primate research program. We thank Buddy Capuano (Wisconsin National Primate Research Center) and P. Frost (Texas Biomedical Research Institute) for excellent veterinarian oversight of our research program. We also thank D. McFarland (formerly Pittsburgh) for expert technical assistance as well as Oregon Primate Research Center and the Texas Biomedical Research Institute for monkeys utilized during this study. We thank Bruce Campbell and Angela Palermo Lauff for expert editorial assistance and manuscript submission. Past NIH support to G.S. is gratefully acknowledged.

\section{Author contributions}

C.R.S. and G.S. designed the experiments and analyzed the data. C.C., C.N. and E.J. performed laparoscopic oocyte retrieval and collections and prepared fresh sperm samples for fertilization experiments. C.R.S., D.T. and L.H. performed IVF and ICSI fertilization of nonhuman primates. E.J., D.T. and C.R.S. conducted the live time-lapse imaging, analyzed data, and archived the datasets. C.R.S. and C.H. prepared immunocytochemistry data with data analysis and prepared final figure plates and videos for submission. G.S. and C.R.S. wrote the manuscript. All authors reviewed and approved the final manuscript.

\section{Competing interests}

The authors declare no competing interests.

\section{Additional information}

Supplementary information is available for this paper at https://doi.org/10.1038/s41598-019-51815-4.

Correspondence and requests for materials should be addressed to G.S.

Reprints and permissions information is available at www.nature.com/reprints.

Publisher's note Springer Nature remains neutral with regard to jurisdictional claims in published maps and institutional affiliations.

(c) (i) Open Access This article is licensed under a Creative Commons Attribution 4.0 International License, which permits use, sharing, adaptation, distribution and reproduction in any medium or format, as long as you give appropriate credit to the original author(s) and the source, provide a link to the Creative Commons license, and indicate if changes were made. The images or other third party material in this article are included in the article's Creative Commons license, unless indicated otherwise in a credit line to the material. If material is not included in the article's Creative Commons license and your intended use is not permitted by statutory regulation or exceeds the permitted use, you will need to obtain permission directly from the copyright holder. To view a copy of this license, visit http://creativecommons.org/licenses/by/4.0/.

(C) The Author(s) 2019 